\section{P1.039 SELECTIVE MODULATION OF B-CELL MARKERS OF ACTIVATION, INHIBITION AND EXHAUSTION WITH VASOACTIVE INTESTINAL PEPTIDE (VIP) IN ASYMPTOMATIC HIV INFECTION}

doi:10.1136/sextrans-2013-051184.0260

'T D Reid, ${ }^{2} \mathrm{H}$ Ipp, ${ }^{3} \mathrm{~L}$ Becker, ${ }^{1} \mathrm{R}$ H Glashoff. 'Division of Medical Virology, Department of Pathology, Faculty of Medicine and Health Sciences, Stellenbosch University, Cape Town, South Africa; ${ }^{2}$ Division of Haematology, Department of Pathology, Faculty of Medicine and Health Sciences, Stellenbosch University \& NHLS, Cape Town, South Africa; ${ }^{3}$ Desmond Tutu Foundation for HIV Research, University of Cape Town, Cape Town, South Africa

Background Chronic HIV-1 infection is characterised by immune activation and exhaustion. This study investigated B-cell subset distribution and novel markers of immune activation and exhaustion in an asymptomatic untreated HIV-infected cohort; and VIP's effect thereon. The immunomodulator, VIP, is known to limit T-cell activation. No studies to date have examined VIP's on B-cell activation in the context of HIV infection.

Methods HIV+ patients and matched HIV- controls were recruited at Emavudleni, a voluntary HIV testing and prevention clinic in Crossroads, Cape Town. B-cells were isolated via RosetteSep enrichment; cultured for $18 \mathrm{~h}$ with either LPS, or R848 alone, or with VIP; and then analysed via BD FACSCanto II. B-cell subsets were as follows: activated memory (AM), resting memory (RM: $\mathrm{CD} 21+\mathrm{CD} 27+)$, mature naïve (MN: CD21+CD27-), or tissue-like memory (TLM: CD21loCD271o). Surface expression of markers of activation (CD126, CD86, CD38, CD284) exhaustion (CD72, CD85j, CD305, CD300a, CD307d), and apoptosis signalling (CD95) were measured. These were also compared to standard markers of immune activation (CD38+CD8 + T-cells) and HIV infection (CD4 count and plasma viral load).

Results RM was decreased, while TLM was increased $(p<0.01)$ with HIV-infection. CD126 \& CD86 expression on AM \& RM decreased by $20 \%$ with VIP inhibition; while AM, RM, TLM \& MN CD72 expression decreased by 63\%. CD85j AM \& TLM expression decreased by $45 \%$, \& CD95 expression on RM, TLM, \& MN decreased by $52 \%$ with VIP inhibition (all $p<0.001$ ).

Conclusion Our data indicated that B-cells are in a more activated state and possibly more prone to apoptosis in untreated, asymptomatic HIV, and that addition of VIP resulted in a near complete downregulation of markers associated with activation, exhaustion, and apoptosis. VIP is a potentially valuable novel immunnomodulatory agent for the limitation of B-cell activation, alleviation of exhaustion and selective modulation of apoptosis in asymptomatic HIV infection.

\section{P1.040 TIM-3 AND PD-1 ARE DIFFERENTLY EXPRESSED ON EXHAUSTED T CELLS IN HIV INFECTED PATIENTS}

doi:10.1136/sextrans-2013-051184.0261

'J Leitner, ${ }^{2} \mathrm{~A}$ Rieger, 'W Pickl, 'G Zlabinger, ${ }^{2} \mathbf{K}$ Grabmeier-Pfistershammer, ${ }^{1} \mathrm{P}$ Steinberger. 'Institute of Immunology, Medical University Vienna, Vienna, Austria; ${ }^{2}$ DIAID, Dermatology, Medical University Vienna, Vienna, Austria

Progressive loss of $\mathrm{T}$ cell function is an important mechanism of chronic HIV-1-infection. PD-1 has been primarily used to describe exhausted $\mathrm{T}$ cells. Recently Tim- 3 has been identified as additional marker for dysfunctional T cells. Tim-3 positive cells have not been defined in detail.

In this study we investigated the expression of PD-1 and TIM-3 on $\mathrm{T}$ cells from HIV-1-infected individuals.

We found that in the viremic patients only a small percentage of $\mathrm{T}$ cells expressed Tim-3 (mean: 4.1\%). In contrast, a significant amount of T cells were PD-1 positive (mean 36.8\%) and PD-1 was expressed at much higher levels than Tim-3. Nevertheless we found a trend to higher numbers of Tim-3 positive cells in viremic than in aviremic
HIV-infected and even lower numbers in healthy individuals. When analysing CD8 $\mathrm{T}$ cells regarding CD45RA expression we found a striking difference between Tim- 3 and PD-1 positive T cells: Tim-3 expressing cells were found in the CD45RA positive subset $(p<0.5)$ whereas PD-1 expression was nearly exclusively found on the CD45RA negative subset $(p<0.5)$. To further characterise the Tim-3 positive subset we performed multicolour staining using antibodies to various $T$ cells markers including CD28 and CD57. Although CD57 expression and loss of the CD28 molecule are both associated with T cell senescence, Tim-3 expressing cells were exclusively found in the CD57 positive subset whereas is was not restricted to CD28 negative $T$ cells.

In contrast to the PD-L/PD-1 pathway, blockingTim-3 did not enhance HIV-specific T cell proliferation or IFN g secretion.

Furthermore, whereas PD-1 positive cells have previously been shown to be increased in HIV-infected patients with discordant immune response, Tim- 3 expression did not differ between patients with or without immune restoration on HAART.

Taken together our data implicate that Tim-3 defines a novel subset of terminally differentiated $\mathrm{T}$ cells.

\section{P1.041 HIV DRUG-RESISTANCE MUTATIONS WITHIN HIV REVERSE- TRANSCRIPTASE AMONG PATIENTS RECEIVING HAART IN KAZAKHSTAN}

doi:10.1136/sextrans-2013-051184.0262

A Utegenova, N Dzissyuk. Republican centre for prevention and control of AIDS, Almaty, Kazakhstan

Introduction Drug resistance is very serious clinical problem, it is often cause of treatment failure among HIV-positive patients on HAART. Antiretroviral therapy (ART) was started in 2005. The aim of this study is to analyse of the prevalence of drug-resistance mutations (DRMs) within reverse-transcriptase (RT) among HIV-positive on first-line ART.

Methods 205 HIV-positive patients on (AZT/3TC + NVP or EFV) with viral load more than $1500 \mathrm{c} / \mathrm{ml}$, and 37 ART-naïve were examined. HIV RNA extraction, RT-PCR and sequencing pol gene were assayed with the kits "ViroSeq HIV-1 genotyping systems" (USA) or "AmpliSens-HIV-genotype" (Russia). Sequencing was performed on ABI3130. To interpret results the programme of the Stanford University (www.hivdb.stanford.edu) was used. HIV subtyping was performed using "REGA HIV-1/2" (www.bioafrica.net), "Comet HIV 1/2" (www.comet.retrovirilogy.lu).

Results Among the received RT sequences HIV-1 subtypes A(A1) (71.1\%), CRF02 AG (25.0\%), B (2.9\%), CRF03 AB (0.5\%), сpx06 $(0.5 \%)$ were identified. Overall, $34.6 \%$ (71/205) patients' treatment failure due to drug-resistance virus. In $22.5 \%$ cases were detected drug-resistance mutations (DRMs) to both NRTI and NNRTI. There was no correlation between the formation of drug resistance and the HIV-1 subtype A, B and A/G (35.9\%, 33.3\% and $37.3 \%$, respectively).

In the NRTI group were identified M184V, T215F, and K219O mutations. Among DRMs to NNRTI G190A/S, K103N, K101E/H and Y181C/V mutations were registered. Among ART-naïve patients DRMs was not detected.

Conclusion In Kazakhstan the HIV resistance was determined in $3.9 \%$ among HAART treated patients and mostly to 3TC, NVP and EFV. It's necessary to increase the variety of antiretroviral drugs used in the country.

\section{P1.042 WITHDRAWN BY AUTHOR}

P1.043 TRICHOMONAS VAGINALIS IN A MACACA NEMESTRINA MODEL: EVALUATING AGE AND DETECTION ASSAYS

doi:10.1136/sextrans-2013-051184.0263

Y Cosgrove Sweeney, K J Agnew, D L Patton. U of WA, Seattle, WA, United States 